\title{
CIRED 2013 - Internationale Konferenz über elektrische Verteilnetze
}

\author{
H. Haidvogl
}

Online publiziert am 30. November 2013

(c) Springer Verlag Wien 2013

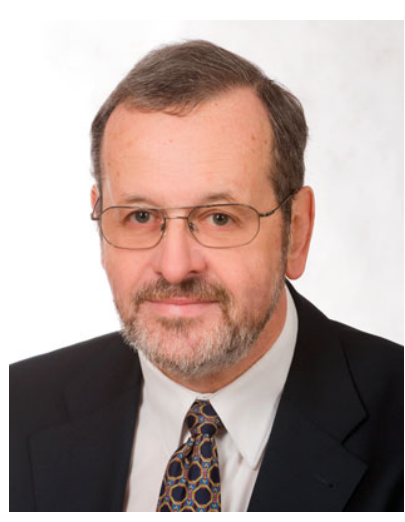

Prok. Dipl.-Ing. Herbert Haidvogl
Die 22. Konferenz fand vom 10. bis 13. Juni 2013 im Tagungszentrum Kistamässan in Kista bei Stockholm, Schweden, statt. Rund 1100 Teilnehmer aus 56 Ländern der ganzen Welt nahmen an der Veranstaltung teil. Die UNSanktionen gegen Iran und die unsichere Lage in Nordafrika sowie im arabischen Raum ließen die Zahl auf das Niveau von 2009 sinken. Die sechs Session Chairmen und elf Rapporteure, davon zwei aus Ös-

terreich, hatten die Aufgabe, rund 1500 Beitragsvorschläge zu lesen und zu beurteilen. Aus Österreich wurden 39 Beiträge eingereicht, eine sehr erfreuliche Zahl. Schließlich wurden 680 Beiträge, davon 22 aus unserem Lande, für die Präsentation während der Konferenz ausgewählt.

Das gewohnt breitgefächerte Angebot für die Konferenzbesucher umfasste:

- 6 Haupt-Sessionen mit 140 Vorträgen, davon acht aus Österreich, inklusive Diskussionen

- 14 Round Table-Diskussionen, davon zwei unter österreichischer Leitung

- zahlreiche Führungen durch die sechs Poster-Sessionen mit Präsentationen durch die Autoren (davon 14 aus Österreich)

- 6 Forschungs- und Innovationsforen (RIF) - mit vier Beiträgen aus Österreich

- 6 Tutorials - für Jungingenieur/inn/e/n

- eine Fachausstellung mit 86 Anbietern, davon drei aus Österreich

Die Konferenz begann mit dem Opening Forum und behandelte die Rolle der Verteilnetze in Europa und in Schweden sowie Entwicklungstrends der Stromversorgung in China. An diesem Abend wurde auch der Abschlussbericht der CIRED-Arbeitsgruppe (AG) "Smart Grid" durch den Vorsitzenden Prof Dr. Markus Zdrallek, Bergische Uni Wuppertal, Deutschland, präsentiert. In dieser AG hatte Dipl.Ing. Helfried Brunner, AIT, intensiv mitgearbeitet. Der Schlussbericht steht unter der Webadresse http://www.cired.net/files/download/65 gratis zum Download zur Verfügung.

Die dreitägige Konferenz wurde mit einem Galadinner am Mittwochabend ergänzt. Die Teilnehmer erlebten im Wasa-Museum eine spektakuläre Perfomance Show an den Museumswänden über ihren Köpfen. Die Tische waren rund um das 1961 gehobene und aufwändig restaurierte Kriegsschiff Wasa, welches bei seiner Jungfernfahrt am 10. August 1628 gleich im Hafen von Stockholm gesunken war, angeordnet. Der weitere Abend bot Köstlichkeiten der schwedischen und internationalen Küche, begleitet von ABBA-Musik einer Live Band.

Am Freitag gab es die Möglichkeit, technische Einrichtungen in Stockholm und Umgebung kennen zu lernen.

Die CIRED 2013 besuchten 47 Delegierte aus Österreich (7 weniger als 2011). 22 heimische Beiträge (3 weniger als 2011) wurden diskutiert. Dieses erfreuliche Ergebnis stärkt die Position Österreichs in der internationalen CIRED-Gemeinschaft.

\section{An den CIRED-Info-Nachmittagen}

am 28. Jänner 2014, 14 Uhr, in den Räumen von Österreichs Energie, 1040 Wien Brahmsplatz 3 sowie

am 4. Februar 2014, 14 Uhr, im Hotel Hilton, 6010 Innsbruck, Salurner Straße 15, werden Experten des österreichischen Nationalkomitees über aktuelle Trends von der CIRED 2013 Konferenz berichten und Ihre Fragen gerne beantworten.

Die nächsten internationalen CIRED-Veranstaltungen sind folgende:

- 4. Workshop am 11. und 12. Juni 2014 in Rom zum Thema "Challenges of implementing active distribution system managment" http://www.cired2014-workshop.org sowie die

- 23. CIRED-Konferenz vom 15. bis 18. Juni 2015 in Lyon, Frankreich http://www.cired2015.org

Ich lade Sie wieder herzlich ein, mit Fachbeiträgen Ihre Erfahrung international mit anderen zu teilen und von anderen Teilnehmern zu profitieren. Gerade für junge Ingenieure ist CIRED die ideale Plattform, internationale Erfahrung in der Präsentation und Diskussion in Englisch zu sammeln.

Am Directing Comittee Meeting in Stockholm wurden Dipl.-Ing. Theodor Connor, Siemens-Erlangen, Deutschland, zum Chairman des Directing Committee, und Prof. Emmanuel de Jaeger, Université catholique de Louvain, Belgien, zum Chairman des Technischen Komitees gewählt.

Über das österreichische Nationalkomitee ist zu berichten, dass es 24 Mitglieder und vier Ehrenmitglieder aufweist. CIRED Österreich ist ein Teil der OGE Österreichische Gesellschaft für Energietechnik im OVE Österreichischer Verband für Elektrotechnik. Aktuelle Neuigkeiten können der CIRED-Homepage unter http://www.cired.at entnommen werden.

Bei der internationalen CIRED sind zwölf Experten aus Österreich aktiv. Diese sind die Herren Ao. Univ.-Prof. Dr. Herwig Renner, Institut für Elektrische Anlagen der TU Graz, als neu bestellter Chairman der Session 2, Dipl.-Ing. Ignaz Hübl, Bereichsleiter KELAG als Rapporteur in der Sessions 3, Dipl.-Ing. Dr. Gerhard Jambrich, Bereichsleiter BEA Electric, und Dipl.-Ing. Herwig

Haidvogl, Herbert, Netz Niederösterreich GmbH, EVN-Platz 1, 2344 Maria Enzersdorf, Österreich (E-Mail: Herbert.Haidvog|@netz-noe.at) 


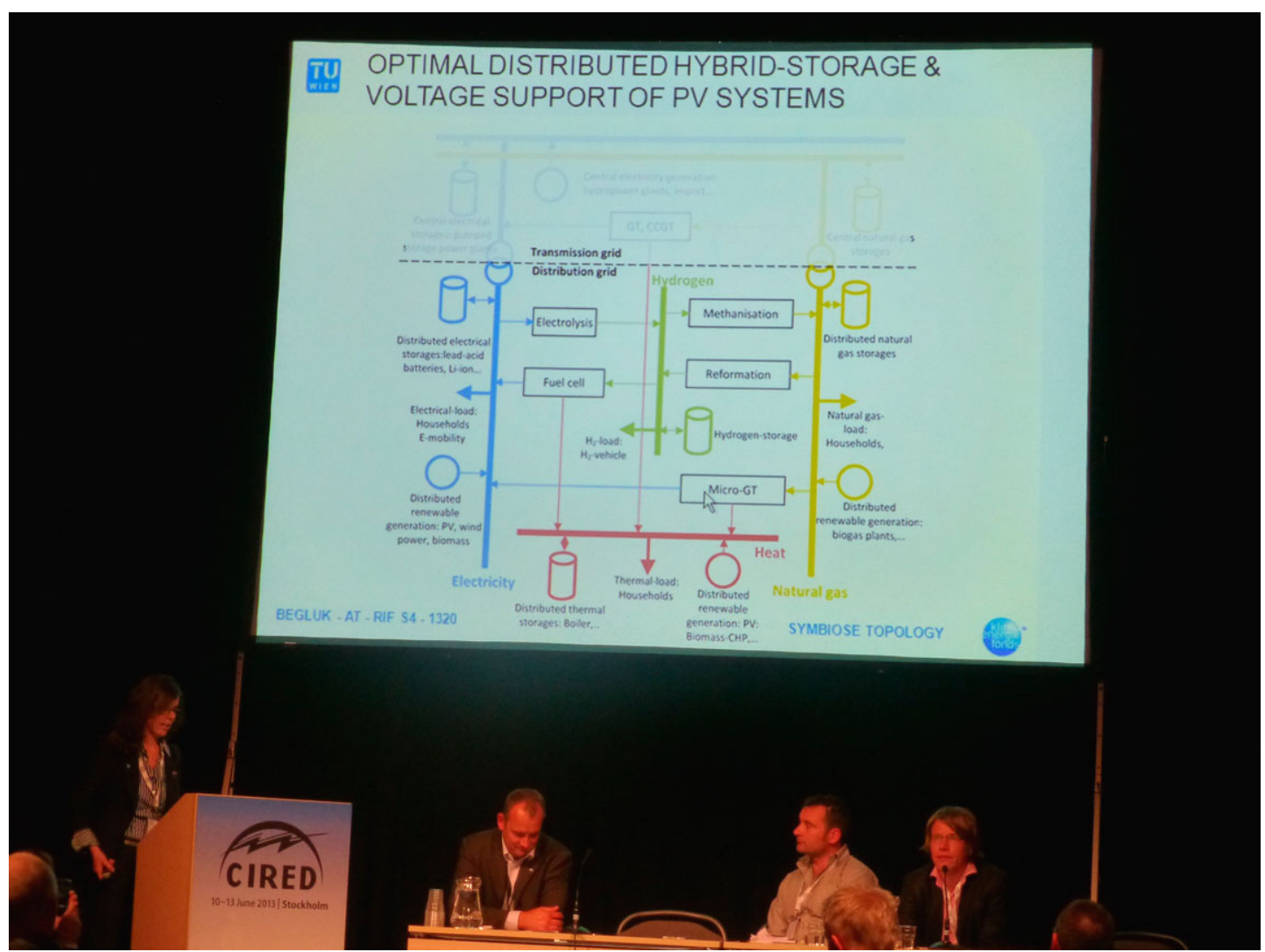

Präsentation eines Beitrags der TU Wien

Struber, MBA, Bereichsleiter Salzburg Netz GmbH, sind Mitglieder in den Sessions Advisory Groups 1 und 6. Univ.-Prof. Dr. Lothar Fickert, TU Graz und Dipl.-Ing. Werner Friedl, E-Control, waren wesentlich an den Round Table-Diskussionen in Stockholm beteiligt.

Die Herren Univ.-Prof Dr. Wolfgang Gawlik, TU Wien, Dipl.-Ing. Dr. Manfred Wurm, Netz NÖ, und Dipl.-Ing. Helmut Sprongl, E-Control, arbeiten seit Frühjahr 2013 in der JWG CIGRE-CIRED mit.

An der Gründung der Gemeinsamen Arbeitsgruppe (JWG) CIGRECIRED über "Adaptation of Earthing Systems to Reality Related Requirements" ist Prof. Dr. Lothar Fickert maßgeblich beteiligt.

Weitere österreichische Experten sind herzlich eingeladen, das internationale CIRED-Team zu verstärken. Darüber hinaus bietet sich künftig die Chance, Know-how in weiteren AG bzw. Workshops einzubringen und zu bekommen, welche in Kooperation mit CIGRE und EURELECTRIC geplant sind

Herzlichen Dank an alle Beteiligten für die geleistete Unterstützung. Ich wünsche als scheidender Vorsitzender der CIRED frucht- bringende Diskussionen und interessante Veranstaltungen mit hoher internationaler österreichischer Beteiligung.

Als Ansprechpersonen für Fragen zu CIRED stehen Ihnen gerne zur Verfügung:

Assistenz CIRED Österreich: Frau Elisabeth Schmidbauer, LLB.oec. LLM.oec., Assistenz Salzburg Netz, E-Mail: elisabeth.schmidbauer@salzburgnetz.at

Geschäftsführer CIRED-Österreich: Herr Dipl.-Ing. Herwig Struber, MBA; Bereichsleiter Salzburg Netz, E-Mail:

herwig.struber@salzburgnetz.at

Designierter Vorsitzender NK CIRED Österreich: Herr Dipl.-Ing. Dr. Walter Tenschert; Geschäftsführer Netz OÖ, E-Mail:

walter.tenschert@netzgmbh.at

Mögen die anschließenden Expertenberichte über die CIRED 2013 eine anregende Lektüre sein. 